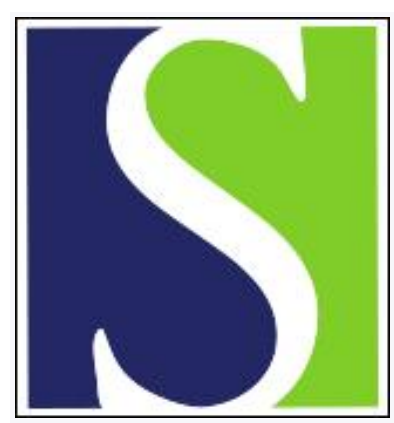

Scand J Work Environ Health 1996;22(6):401-404

https://doi.org/10.5271/sjweh.160

Issue date: Dec 1996

Exposure assessment -- a challenge for occupational epidemiology

by Kauppinen $T$

This article in PubMed: www.ncbi.nlm.nih.gov/pubmed/9000306

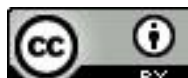

This work is licensed under a Creative Commons Attribution 4.0 International License 


\section{Exposure assessment - a challenge for occupational epidemiology}

The problems of exposure assessment have been tackled in many scientific meetings on occupational epidemiology. Milestones in this field are the special symposiums held in Woods Hole (1), Leesburg (2) and Lyon (3). This issue of the Scandinavian Journal of Work, Environment \& Health includes an article in which Stewart, Lees \& Francis review the process of exposure assessment in cohort studies from the selection of agents to the validation of the assessment methods.

Why does exposure assessment interest epidemiologists? There are several reasons. First, the exposure-response relation is replacing simple hypothesis testing as the key object of epidemiologic studies, and it requires quantitation of exposures instead of qualitative categorization. Second, quantitative risk assessment at the individual or community level requires reliable information on exposures. Third, many higher risks have already been found, and the detection of lower risks requires valid design, reliable methods in exposure assessment, and careful control of confounding factors. Finally, the increasing amount and computerization of monitoring data on exposure enables the use of more sophisticated and effective assessment methods than earlier. Occupational epidemiologists are facing the challenge of low and complex exposures, but, on the other hand, the availability of data and recent methodological progress are improving the reliability of exposure assessment. The battle is on.

What is the present state of the art in exposure assessment? From the theoretical point of view, the situation is not very satisfactory. Statistical theories have been applied to assess the effects of misclassification of exposure, to analyze the variability of exposure between and within exposure groups, to derive estimates under different exposure models, and to develop more predictive indices of exposure. All these issues have important implications for the validity of epidemiologic studies. However, the theoretical framework which would anchor exposure assessment to epidemiology is vague, and often exposure issues are still planned and treated as a separate, although necessary, appendix of an epidemiologic study. The conceptual form of the study hypotheses or occurrence relations have in some studies been derived from pharmacokinetic models, which are indeed the most logical basis for inferring the relevant characteristics of exposure to be assessed or measured. Their use is still exceptional however and may also remain unfeasible in many studies. Nevertheless, the characteristics of exposures and the predictive indices of exposure need to be focused on to detect low risks. The article of Stewart and her co-workers discusses this issue through practical examples.

The methods of exposure assessment differ in cohort and case-referent studies. In cohort studies, the most common method is to assess exposure on the basis of jobs held and industrial hygienic data. Job categories are usually treated as homogeneous exposure groups (ie, the same exposure estimate is applied to the whole group). This is a practical approach, but it has turned out that a homogeneous exposure group is often only a myth. Exposure within a homogenous exposure group may vary so much that the resulting misclassification spoils all possibilities to find the exposure-response relation, if there is one. Stewart and her colleagues use a more neutral concept of exposure group and point out that statistical variance analysis of exposure data helps to determine whether the job groups are a solid basis for exposure groups or whether some other factor, such as the product, process or worksite, is more appropriate.

Another common problem in cohort studies is the incompleteness of exposure data. Statistical or deterministic modeling or professional judgment is used to generate the missing data. If an abundance of data is available, statistical modeling is definitely preferable. However, professional judgment should 
never be discarded. Knowledge and experience are needed to assess the representativeness and reliability of data to be used in statistical analysis, and an expert's opinion is the only alternative when exposure data are missing altogether, which is often the case, for example, in community-based casereferent studies. There is a flavor of similar controversy among exposure assessors as among statisticians, who may emphasize either actual data ("frequentists") or a prior view of the assessor, which is based on previous information and one's own insight into the topic ("Bayesians"). It is easy to imagine situations in which exposure data, especially if valid and ample, are undoubtly more accurate than any professional judgment. On the other hand, it is as easy to admit that an experienced assessor can provide better estimates than what biased or unrepresentative data would allow. Perhaps a good practice would be not to use data-based and judgment-based estimates systematically, but to select the estimate judged to be the best case by case. Another matter worth stressing in professional judgments is that, in order to be credible, the definition of exposure and the inferences behind the estimates need to be accurately documented - the black box of the exposure assessor should be illuminated so that the validity of estimates can be assessed. Documentation is necessary also when exposure information is being shared at the national and international levels; this is a highly recommendable way to improve the quality of professional judgments.

Job-exposure matrices can be used as semiautomatic tools of exposure assessment in several types of studies. In spite of hard criticism, job-exposure matrices have not turned out to be a track of errors in the history of occupational epidemiology. In very large studies, whether of the cohort or case-referent design, the job-exposure matrix approach is often the only feasible way to assess exposure. But there are differences between job-exposure matrices. Their structure varies widely, as does their validity from nil to perfect. It is therefore important that the users know how to use the job-exposure matrix and how valid it is for the specific exposure(s) under study. The traditional job-exposure matrices which crudely specify exposure in qualitative (exposed, unexposed) or ordinal (low, medium, high) terms without any further documentation are losing their function, along with the requirements for better quality. Future job-exposure matrices will probably be more like exposure information systems which are quantitative and include accurate definitions and documentation. They can provide quantitative proportions and levels of exposure within occupations or other exposure groups, estimates on the numbers of exposed workers, descriptions of sources of exposure, relevant monitoring results, and bibliographic references, which make them useful also outside etiognostically oriented epidemiology in areas such as quantitative risk assessment and hazard surveillance.

The principal problem of exposure assessment is misclassification of exposure, which may mask the true risks. There are many factors which increase exposure misclassification: lack of and unrepresentativeness of exposure data, an inappropriate exposure indicator or its measure, incorrect time reference of exposure, insufficient exposure contrast in the study base, omission of confounding exposures, to name a few. All these complicate the efforts of epidemiologists to find the true risk and exposureresponse relation which tend to vanish in the horizon like a mirage. Our understanding of the effects and interplay of these factors is poor. A computer program which would simulate the observed exposureresponse relation, when the true relation, structure of the study base, temporal exposure pattern and other biasing factors can be varied, would considerably help epidemiologists in the future to predict whether the planned design and study base are powerful enough to detect the potential risks under study. Currently there are many sophisticated statistical packages for analyzing the data of epidemiologic studies but (to my knowledge) none for designing a valid study.

The future will also reveal new problems to make the life of an epidemiologist and exposure assessor still more difficult. Occupation will probably be less accurate as a descriptor of exposure. An increasing portion of the work force will shuttle between different jobs, unemployment, and training periods. Occupational histories and exposures will be ever more complex, varying, and difficult to trace. This 
development will gradually erode the concepts of "occupation" and of "exposure group," and thereby the traditional backbone of occupational epidemiology.

Who will win the on-going battle: the epidemiologist, with his or her powerful computers and exposure data, or reality in all its complexity? Probably sometimes one, sometimes the other, but only the future can reveal the final winner. However, it seems evident that most of the high, easily detectable risks have already been detected, and a valid study design and reliable exposure assessment are becoming more crucial for occupational epidemiology. The exposure assessors will definitely continue their work to improve methods and to share information. But is that enough? If the answer is no, there are fortunately other, perhaps less frustrating, areas where epidemiologic insight and experience are useful for the prevention of occupational hazards, for example, surveillance, intervention research, quantitative risk assessment, and meta-analysis.

\section{Literature}

1. Rappaport SM, Smith TJ, editors. Exposure assessment for epidemiology and hazard control. Chelsea (MI): Lewis Publishers, 1991. Industrial hygiene science series.

2. Special issue on international workshop on retrospective exposure assessment for occupational epidemiologic studies. Appl Occup Environ Hyg 1991;6 June.

3. Special issue on conference on retrospective assessment of exposures in epidemiology. Occupational hygiene - risk management of occupational hazards 1996; vol $3(1-3): 1-208$.

Timo Kauppinen, PhD

Finnish Institute of Occupational Health

Topeliuksenkatu 41 a A

FIN-00250 Helsinki, Finland 